\title{
Tuning the Carrier Lifetime in Black Phosphorene through Family
}

\section{Atom Doping}

\section{Supporting Information}

Hongli Guo ${ }^{1,2}$, Weibin Chu ${ }^{1,3}$, Qijing Zheng ${ }^{1 *}$ and Jin Zhao ${ }^{1,4,5^{*}}$

${ }^{1}$ ICQD/Hefei National Laboratory for Physical Sciences at the Microscale, and CAS Key Laboratory of Strongly-Coupled Quantum Matter Physics, and Department of Physics, University of Science and Technology of China, Hefei, Anhui 230026, China

2 School of Physics and Technology, Center for Nanoscience and Nanotechnology, and Key Laboratory of Artificial Micro- and Nano-structures of Ministry of Education, Wuhan University, Wuhan 430072, China

${ }^{3}$ Department of Chemistry, University of Southern California, Los Angeles, California 90089, United States

${ }^{4}$ Department of Physics and Astronomy, University of Pittsburgh, Pittsburgh PA 15260, United States

${ }^{5}$ CAS Center for Excellence in Quantum Information and Quantum Physics, University of Science and Technology of China, Hefei 230026, People's Republic of China
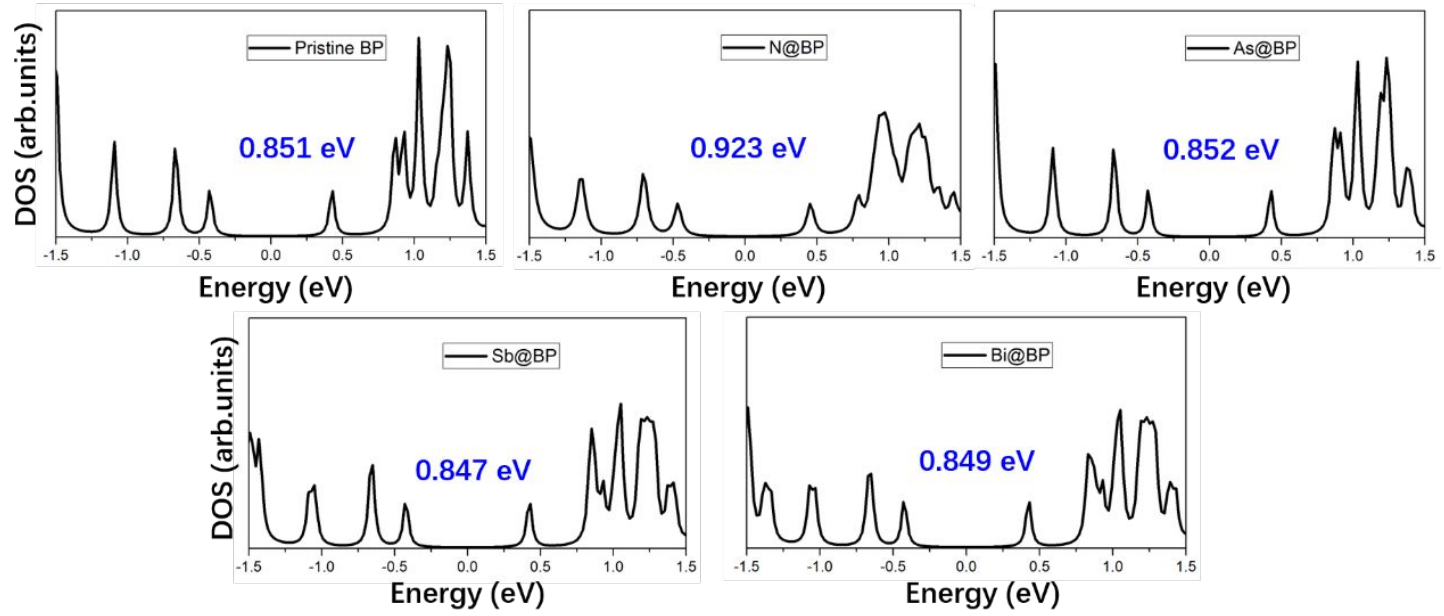

Fig. S1 The density of states (DOS) of pristine and doped black phosphorene obtained by PBE functional. 